\title{
Design and Realization of an Embedded Control Fin Drive
}

\author{
BY \\ Ehab Safwat, A. M. Kamel, Y.Z. El-Halwagy
}

\begin{abstract}
:
The huge developments in embedded systems in addition to its availability for civilian applications in low cost, size and weight attract the researchers in the field of navigation, guidance and control all over the world towards the embedded flight control and autopilots. One of the flight control subsystems is the control fin drive that is responsible of the flying vehicle fins motion control. This paper is devoted to the design and implementation of embedded control fin drive that can be utilized with different guided vehicles such as guided missiles, guided munitions, and unmanned aerial vehicles (UAVs). The design includes calibration of available actuators and feedback sensors in addition to the design of conditioning networks, interfacing and classical controller to stabilize this subsystem and achieve the performance requirements. Also, the design is justified against disturbance and measurement noises. The tests conducted with the designed hardware and the available actuator via the Hardware in the Loop (HIL) simulation show good performance concerning tracking, disturbance rejection and measurement noise. However, the controller necessitates extra tuning to become less sensitive to noises, which is the objective for future work.
\end{abstract}

Keywords: Guidance and Control, Aerodynamics, Mechanics of Flight, Embedded Systems 


\section{Introduction}

The flight control system is a key element that allows the aerospace vehicle to meet its system performance requirements. The objective of the flight control system is to force the aerospace vehicle to achieve the steering commands developed by the guidance system. The types of steering commands vary depending on the phase of flight and the type of interceptor. For example, in the boost phase the flight control system may be designed to force the aerospace vehicle to track a desired flight-path angle or attitude. In the midcourse and terminal phases the system may be designed to track acceleration commands to affect an intercept of the target. The overall stability and performance of the control system are determined by the dynamics of each element in the loop[2].

The missile flight control system is one element of the overall homing loop. A simplified block diagram of the missile homing loop configured for the terminal phase of flight when the missile is approaching intercept with the target is shown in Fig. (1)[1].

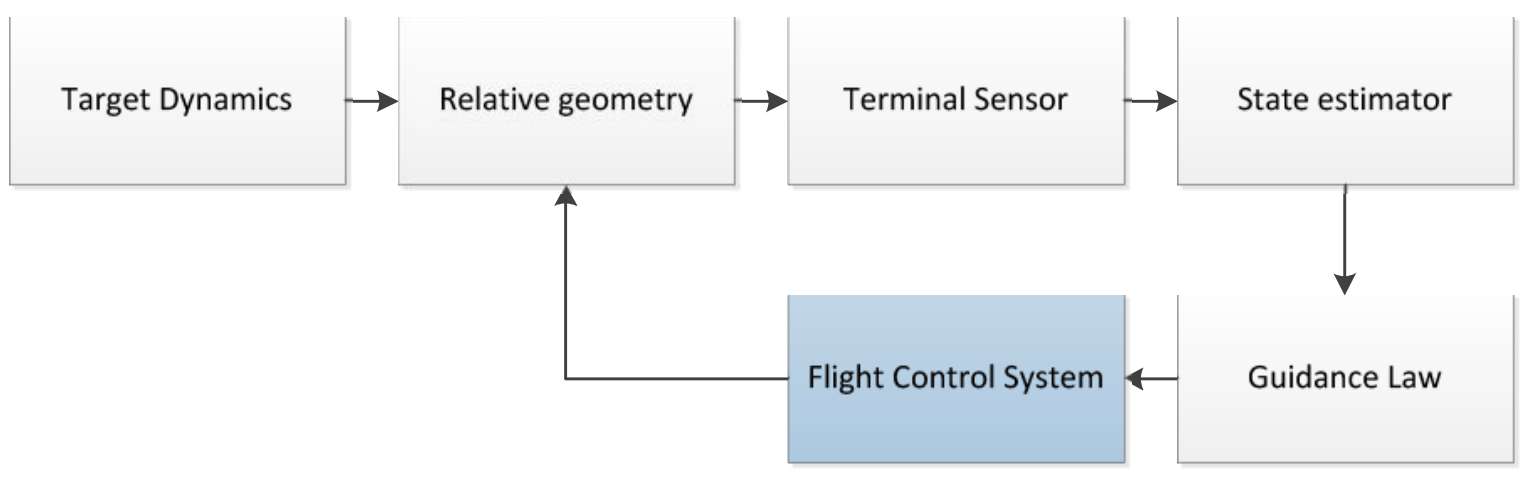

Fig.(1) Missile Homing Loop

The missile and target motion relative to inertial space can be combined mathematically to obtain the relative motion between the missile and the target. The terminal sensor, typically a Radio Frequency (RF) or Infra Red (IR) seeker, measures the angle between an inertial reference and the missile-to-target line-of-sight (LOS) vector, which is called the LOS angle. The state estimator, e.g., a Kalman filter [2], uses LOS angle measurements to estimate LOS angle rate and perhaps other quantities such as target acceleration. The state estimates feed a guidance law that develops the flight control commands required to intercept the target. The flight control system forces the missile to track the guidance commands, resulting in the achieved missile motion. The achieved missile motion alters the relative geometry, which then is sensed and used to determine the next set of flight control commands, and so on. This loop continues to operate until the 
missile intercepts the target. The missile homing loop is a feedback control system that regulates the LOS angle rate to zero. As such, the overall stability and performance of this control system are determined by the dynamics of each element in the loop. Consequently, the flight control system cannot be designed in a vacuum. Instead, it must be designed in concert with the other elements to meet overall homing-loop performance requirements in the presence of target maneuvers and other disturbances in the system, e.g., terminal sensor noise, which can negatively impact missile performance. The flight control system is one element of the overall homing loop. The basic elements of the flight control system, which itself is another feedback control loop within the overall homing loop depicted in Fig.(1) is shown in Fig. (2).

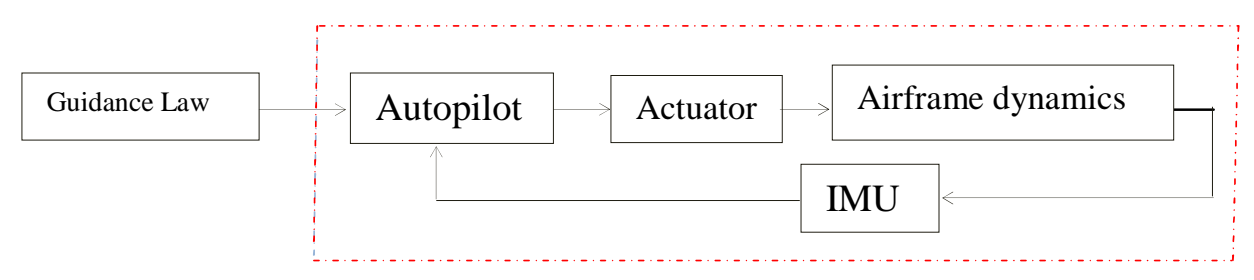

Fig.(2) Automatic Flight Control System

An Inertial Measurement Unit (IMU) measures the aerospace vehicle translational acceleration and angular velocity. The outputs of the IMU are combined with the guidance commands in the autopilot to compute the commanded control input, such as a desired tailsurface deflection or thrust-vector angle. An actuator, usually an electromechanical system, forces the physical control input to follow the commanded control input. The airframe dynamics respond to the control input. The basic objective of the flight control system is to force the achieved aerospace vehicle dynamics to track the guidance commands in a wellcontrolled manner. The inputs to the flight control system are outputs from the guidance law that need to be followed to ultimately hit the target. The specific form of the flight control system inputs (acceleration commands, attitude commands, etc.) depends on the specific application. In general, the flight control system must be designed based on the expected characteristics of the commands, which are determined by the other elements of the homing loop and overall system requirements. Characteristics of concern can be static, dynamic, or both. An example of a static characteristic is the maximum input that the flight control system is expected to be able to track. An example of a dynamic characteristic is the expected frequency content of the command. Other dynamic characteristics of concern 
include the guidance command update rate and the amount of terminal sensor noise flowing into the flight control system and causing unnecessary control actuator activity. The autopilot is a set of equations that takes as inputs the guidance commands and the feedback measurements from the IMU and computes the control command as the output; it must be designed so that the control command does not cause oversaturation of the actuator or the IMU. Because the autopilot usually is a set of differential equations, computing its output involves integrating signals with respect to time. Most modern autopilots are implemented in discrete time on digital computers, although analog autopilots are still used.

Flight control systems are categorized into three main systems namely:

1- Acceleration control system.

2- Attitude control system.

3- Flight path angle control system.

For each system there should be a robust fin drive that can help the missile to achieve its designated task with minimum error.

\section{Actuator (Fin Derive)}

In a control fin drive with position feedback, the control fin deflection $\delta$ is proportional to the magnitude of the control signal i.e. to the required control surface deflection $\delta_{r e f}$ generated from the missile guidance computer. In a control fin drive without or with a rate feedback, the speed of the control fin deflection is proportional to $\delta_{\text {ref }}$. The position feedback is most often realized through a potentiometer, capacitive or induction reader (pickoff) of the control surface deflection. The amplifier has much higher inertia than the servomotor [3]. For simplicity it could be considered as a non-inertial member with amplification $\mathrm{k}_{\mathrm{g}}$. In addition, the negative position feedback is taken as $K_{a}$ to quantify for the hinge moments.

The servomotor transfer characteristics has the following form:

$$
\frac{\delta}{I(s)}=\frac{K_{m} \omega_{n}^{2}}{s^{2}+2 \xi s \omega_{n}+\omega_{n}^{2}}
$$

The stability of the control fin drive can be investigated either algebraically, in case of low order systems, or using any of the known frequency stability analysis methods in conjunction with the computer aided design (CAD) packages such as MATLAB or C. The block diagram of fin drive with position feedback is shown in Fig. (3) 


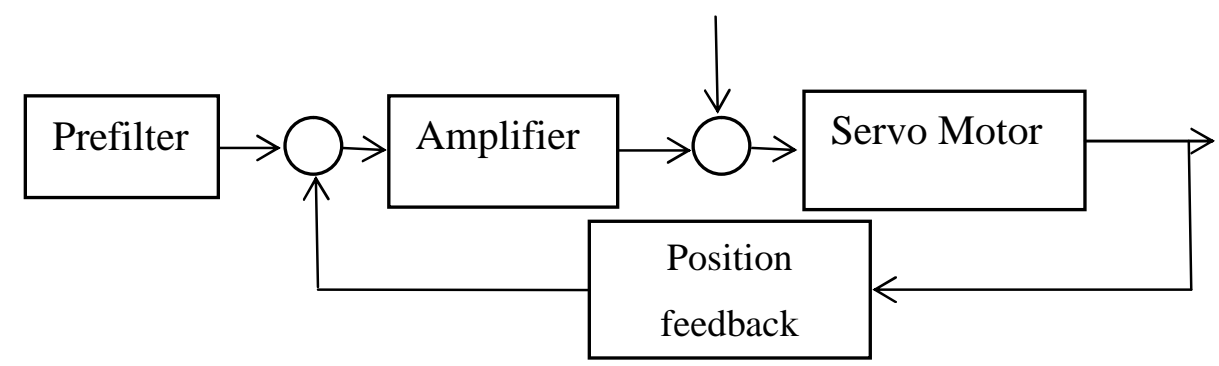

Fig.(3) Block diagram of fin derive

The electric servomotors are part of the control fin drives, especially in small guided missiles.

The control fin servomotor has different properties during missile flight than those during ground functional tests. Therefore, it must have a robust control fin drive to reduce this difference or discrepancy due to the hinge moment. In this paper, a robust control fin drive is designed and implemented as presented in the next sections.

\section{Embedded Systems}

An embedded system is a system in which all its units are assembled to work together according to the plan or program pre-programmed to do a specific function while a general purpose system could be used to run any program of the designer choice. The embedded processor is one component of the electronic system where it is cooperating with the rest of the components to achieve the overall function.

Embedded systems can be classified to:

- Small Scale Embedded System such as single 8 bit or 16bit Microcontroller.

- Medium Scale Embedded System such as Single or few 16 or 32 bit microcontrollers or Digital Signal Processors (DSP) or Reduced Instructions Set Computers (RISC).

- Sophisticated Embedded System with enormous hardware and software complexity.

A Processor is the heart of the Embedded System. There are various types of processors such as:

- General Purpose processor (GPP).

- Microprocessor.

- Microcontroller.

- Embedded Processor. 
- Digital signal Processor.

- Application Specific System Processor (ASSP).

- Multi-Processor System using GPPs.

Nowadays, microcontrollers are hidden inside a huge number of products yielding what is called embedded systems. A microcontroller is a single chip, self-contained computer which incorporates all the basic components of a personal computer on a much smaller scale. Microcontrollers are often referred to as single chip devices or single chip computers. The main consequence of the microcontroller's small size is that its resources are far more limited than those of a desktop personal computer. In functional terms, a microcontroller is a programmable single chip which controls a process or system. Microcontrollers are typically used as embedded controllers where they control part of a larger system such as an appliance, automobile, scientific instrument or a computer peripheral. Microcontrollers are designed to be low cost solutions; therefore using them can drastically reduce part and design costs for a project. Physically, a microcontroller is an integrated circuit with pins along each side. The pins presented by a microcontroller are used for power, ground, oscillator, input/output (I/O) ports, interrupt request signals, reset and control. In contrast, the pins exposed by a microprocessor are most often memory bus signals (rather than I/O ports). Microcontrollers do not function in isolation. As their name suggests they are designed to control other devices. The microcontroller can accept inputs from some devices and provide outputs to other devices within any given system. The microcontroller is often part of a larger system it is often referred to as an embedded controller because it is embedded within the larger system [4].

\section{Embedded fin derive PID Controller}

Tuning a controller on a physical prototype or plant hardware can lead to unsafe operating conditions and damage the hardware. A more reliable approach is to build a plant model and simulate it to verify the controller at different operating conditions so as to run what-if scenarios without risk. The controller must keep the system stable and provide fast reference tracking with minimal steady-state error and overshoot. When modeling is not feasible, the alternative is to develop models from I/O measurements of the plant. System Identification (SI) is then used to obtain the transfer function (Time domain) of Permanent Magnet DC (PMDC) motor platform using the captured I/O data as the input to the SI module. DC Motor parameters are tuned to match the DC motor on the platform, using the 
captured I/O data. The system identification carried out using MATLAB/Simulink SI toolbox.

The parameters of the PID controller are then tuned to control the speed of the DC motor model in the closed-loop structure to track the reference position. The tuned PID controller parameters are then loaded into the microcontroller platform to verify the position response of the DC motor in the hardware platform. The controller must keep the system stable and provide fast reference tracking with minimal steady-state error and overshoot [5].

A potentiometer is used to measure the angle of rotation of the motor shaft, and subtracted from the desired one which is also an analog; the error between them is applied to the controller. The output of the controller is applied to be the duty cycle of the PWM signal. The Pulse-width modulation (PWM) of a signal or power source involves the modulation of its duty cycle, to either convey information over a communications channel or control the amount of power sent to a load. Pulse-width modulation uses a square wave whose pulse width is modulated resulting in the variation of the average value of the waveform. If we consider a square waveform $f(t)$ with a low value $y_{\min } \square$, a high value $y_{\max }$ and a duty cycle $D$, shown in Fig.(4). The average value of the waveform is given by

$$
\bar{y}=\frac{1}{T} \int_{0}^{T} f(t) d t
$$

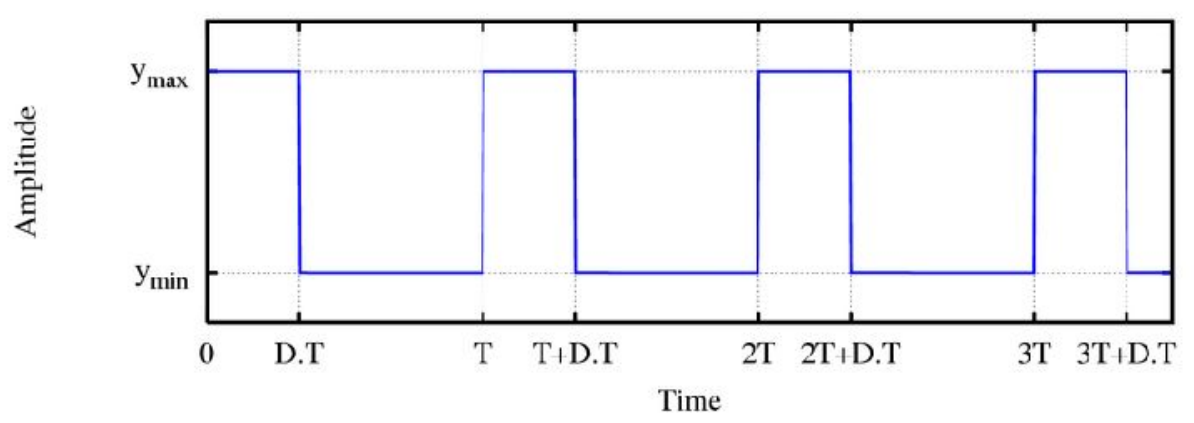

Fig.(4) Square pulse

As $f(t)$ is a square wave, its value is $y_{\max }$ for $0<t<D . T$ and $y_{\min }$ for $D . T<t<T$ The above expression then becomes

$$
\begin{array}{ll}
f(t)=y_{\max } & 0<t<D . T \\
f(t)=y_{\min } & D . T<t<T
\end{array}
$$

Then,

$$
\bar{y}=\frac{1}{T} \int_{0}^{T} f(t) d t
$$




$$
\begin{gathered}
\bar{y}=\frac{1}{T}\left(\int_{0}^{D \cdot T} f(t) d t+\int_{D \cdot T}^{T} f(t) d t\right) \\
\bar{y}=\frac{1}{T}\left(\int_{0}^{D . T} y_{\max } d t+\int_{D \cdot T}^{T} y_{\min } d t\right) \\
\bar{y}=\frac{1}{T}\left(y_{\max D \cdot T}+y_{\min (D \cdot T-T)}\right) \\
\bar{y}=\left(y_{\max D}+(1-D) y_{\min }\right)
\end{gathered}
$$

This latter expression can be fairly simplified in many cases where $\mathbf{y}_{\min }=\mathbf{0}$ as $\overline{\mathbf{y}}=y_{\max } D$. From this, it is obvious that the average value of the signal $(\overline{\mathbf{y}})$ is directly dependent on the duty cycle $D$.

The motor driver integrated circuit (IC) increases the current capability and can drive the motor in both directions. The control signal (PWM signal) from the microcontroller is in TTL level, so it must be amplified to be sufficient for actuator rotation in both directions.

The overall system design that integrated with the microcontroller is shown in Fig.(5).

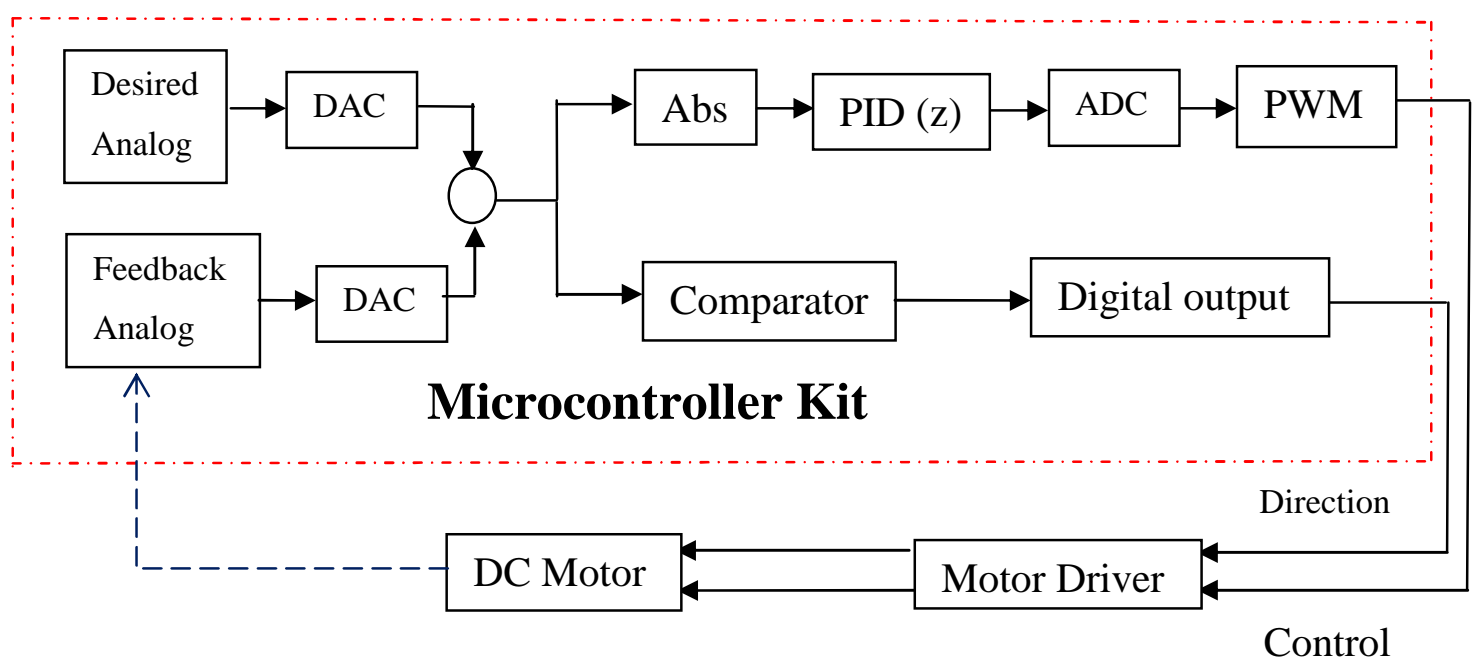

Fig. (5) PID Controller

The feedback signal coming from potentiometer is ranged from -10 volt to +10 volt, but the microcontroller KIT can deal only with TTL signal ranged [0:5] volt. Therefore, a matching circuit is required for the feedback signal to be adapted and acquired by the microcontroller. The feedback signal and it's rescaled version is shown in Fig.(6). 


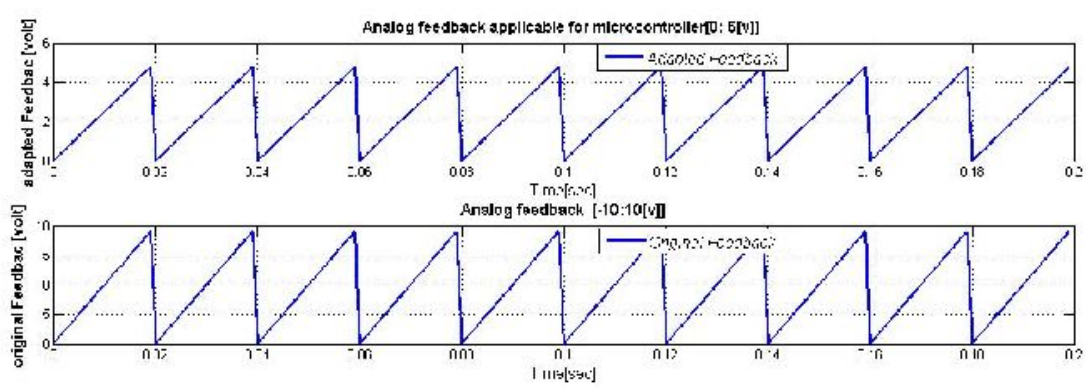

Fig.( 6) Feedback signal

\section{Experimental results}

The error between the desired position and the feedback position is applied directly to the PWM port which analog signal is applied to the driving circuit to initiate the motor to overcome the error .The step response without controller is shown in Fig.(7). In this figure, a motor one turn which corresponds to 365 degrees is commanded. Although the error applied directly to the PWM pin which also produce power according to the value of the duty cycle, but there is overshooting and slow response. The control effort is calculated at the digital output of the error and is shown in Fig.(8).

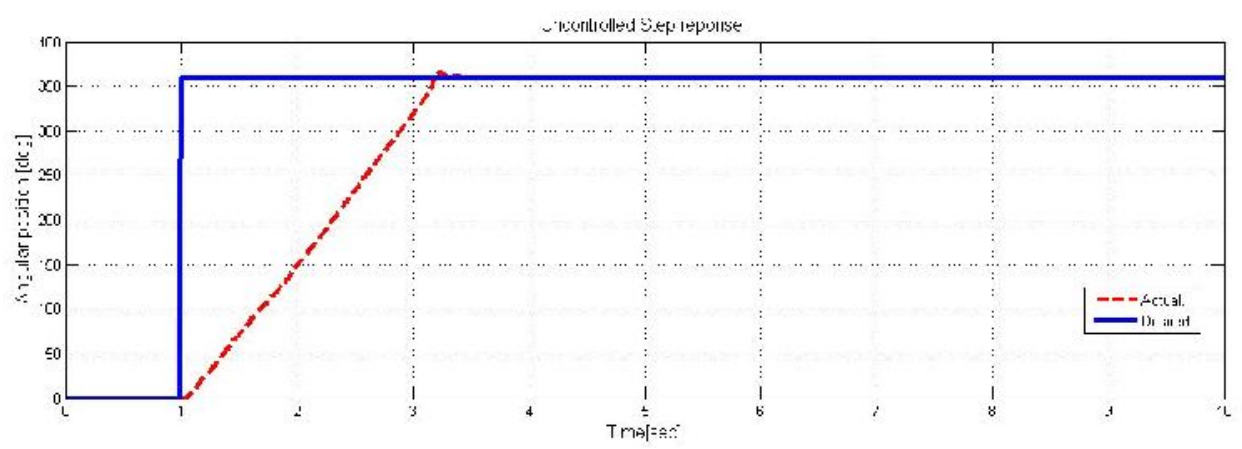

Fig. (7) Uncontrolled Step Response

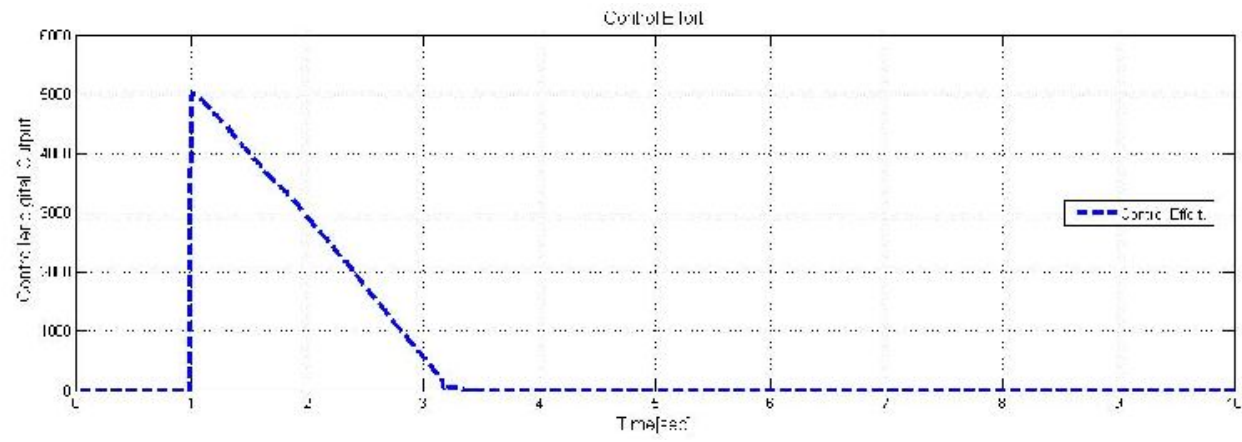

Fig.(8) Control Effort for step input

The experiment is repeated after applying the generated error to a PID controller with following transfer function: 


$$
u(z)=\left(k_{p}+k_{i} \frac{T_{z}}{z-1}+k_{d i} \frac{1}{T_{f}+T_{s} / z-1}\right) e(z)
$$

Where $e(z)$ is the digitized error signal, $\left\{k_{p}, k_{i}, k_{d}\right\}$ are the PID gains. Because of the presence of the derivative term in the control action, the manipulated variable $\mathbf{u}(t)_{\text {Will }}$ involve an impulse function. In an actual PID controller, instead of the pure derivative we use filter of first order to avoid what is called set point kick, so the manipulated variable u(t) will involve a sharp pulse function [5]. The step response is shown in Fig.(9).That enhances the response due to the controller. The resultant control effort is also shown in Fig.(10).

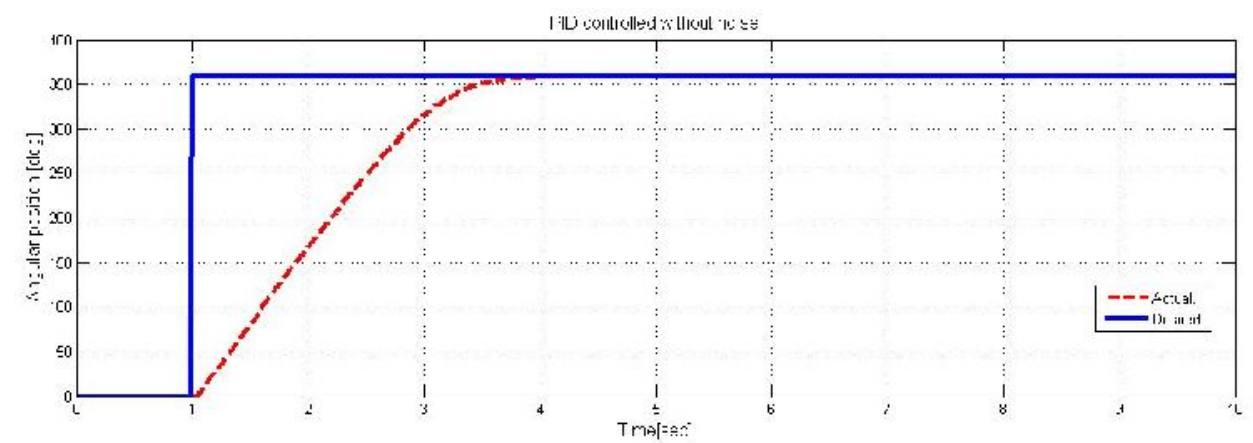

Fig.(9) Controlled step response

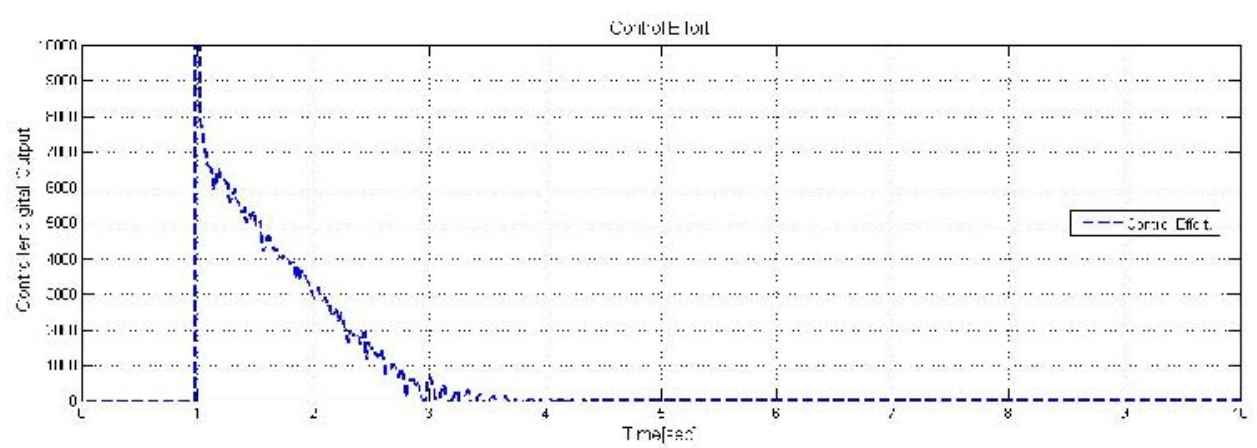

Fig.(10) Control effort for step input without noise

Since one of the PID controller requirements is to reject measurement noise and attenuate the disturbance the step response in the present of Band-Limited White Noise is shown in Fig.(11). The resultant control effort is shown in Fig.(12). 


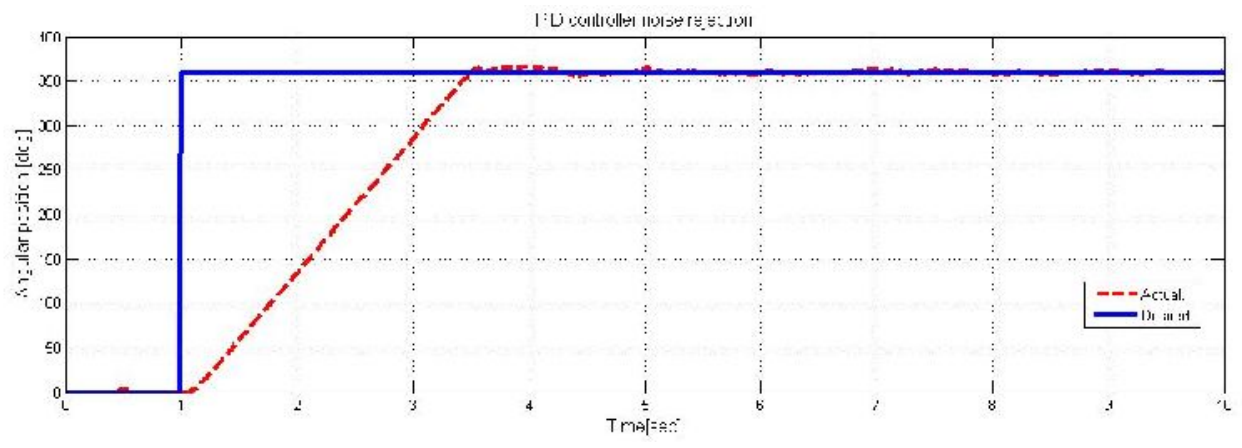

Fig.(11) step response in the present of noise

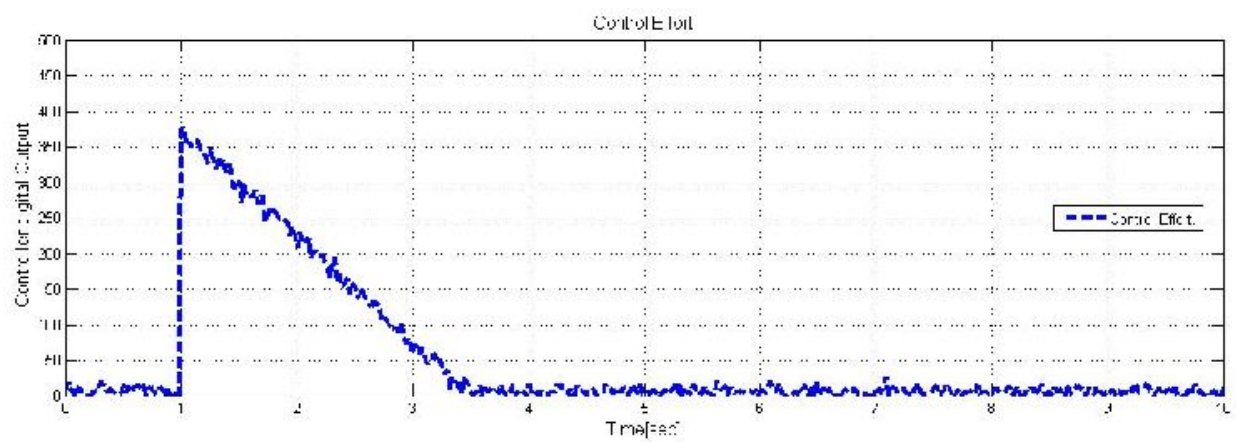

ig.(12) Control Effort for step input without noise

Another test is applied where the motor is manually prevented to move for a short period of time by adding physical obstacles. The step response in the presence of this applied disturbance is shown in in Fig.(13), and the resultant control effort is as shown in in Fig.(14).

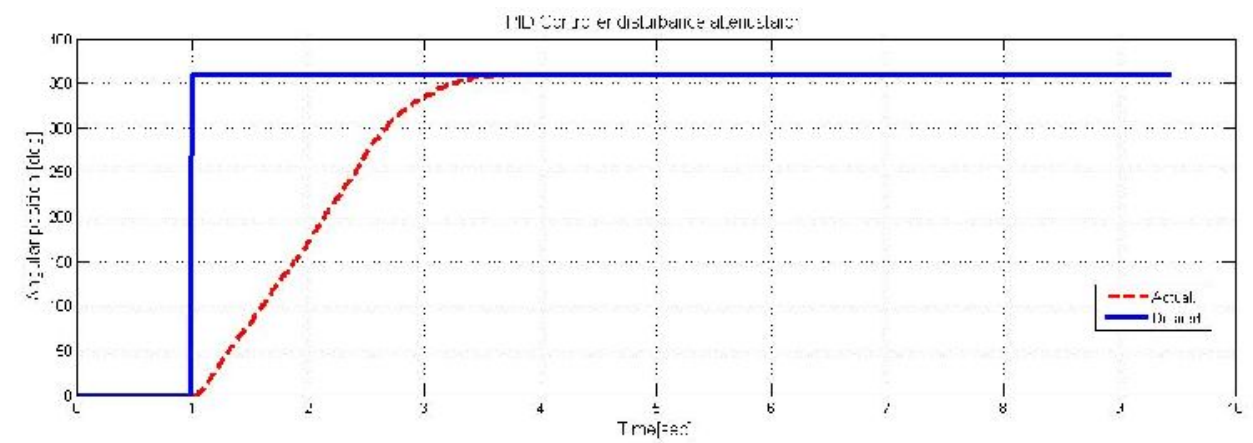

Fig.( 13) Step response in the presence of disturbance 


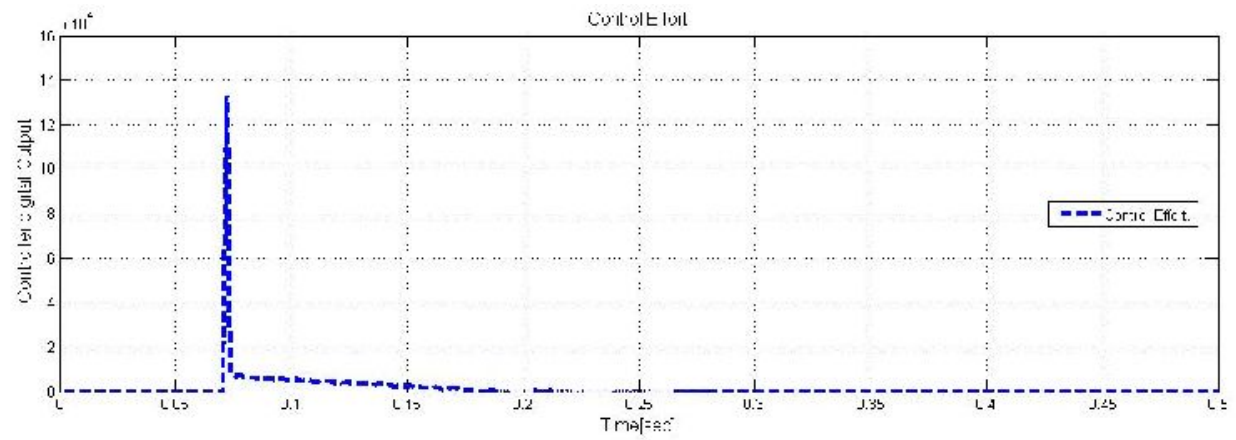

Fig.( 14) Control effort in the presence of disturbance

The performance of the system is limited by the performance of the inner-most loop. The performance of the inner-most loop is often limited by saturation constraints. For example, in the design of the lateral autopilot, the fact that the ailerons have physical limits on their angular deflection implies that the roll rate of the aircraft is limited. Because it is required to simulate the fin motion, the response due to train of pulses that imitates the fin deflection from and to its limits is applied with high frequency. The first test is applied without the designed controller and its response is shown in Fig.(15).

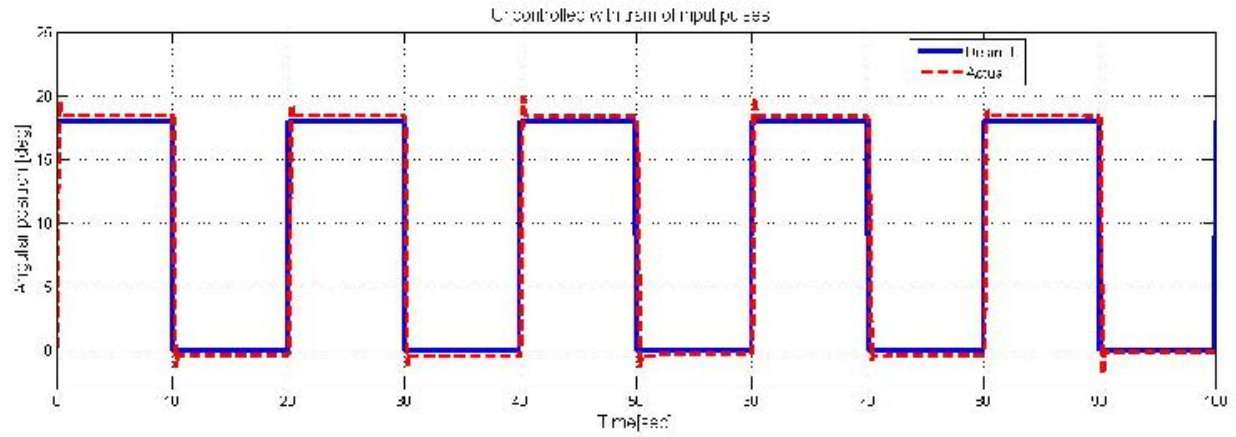

Fig.(15)Uncontrolled with pulse input

The response at the abrupt change has an overshoot and undershooting of about $0.05 \%$ since the error applied directly to the PWM pin without shaping by a controller. The control effort is shown in Fig.(16).

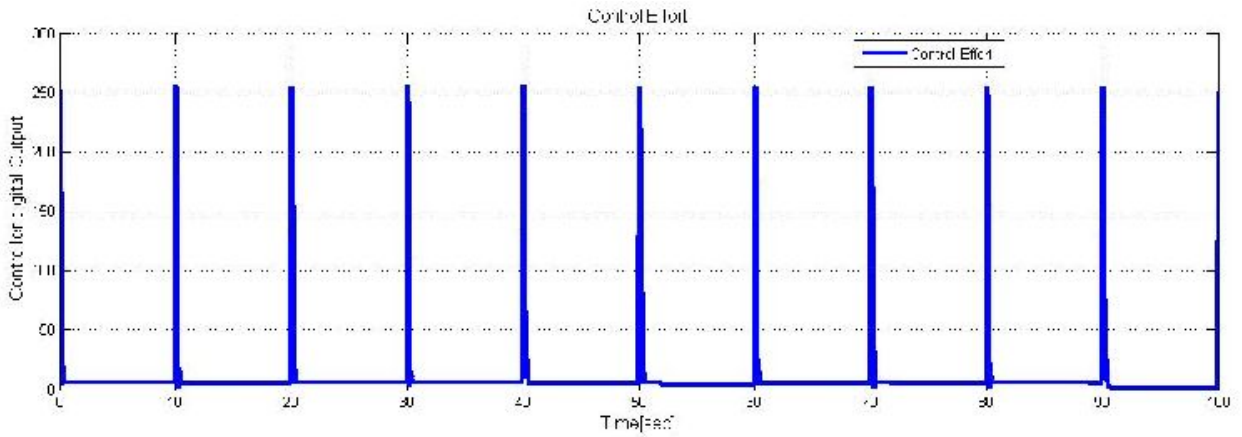

Fig.(16) Control Effort for pulse input 
The response due to train of pulses applied with high frequency with PID controller is shown in Fig.(17) and the control effort is shown in Fig.(18).

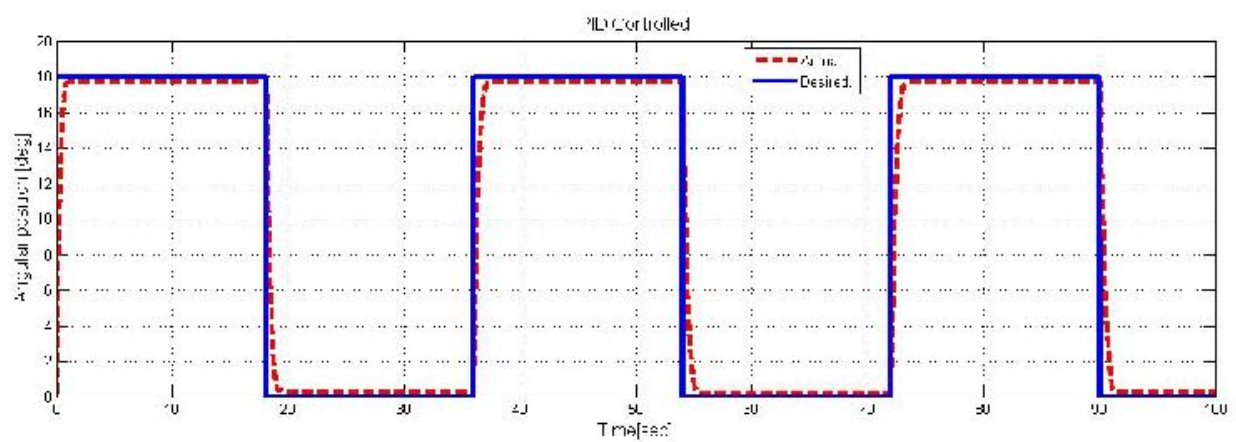

Fig.( 17) Controlled pulse response

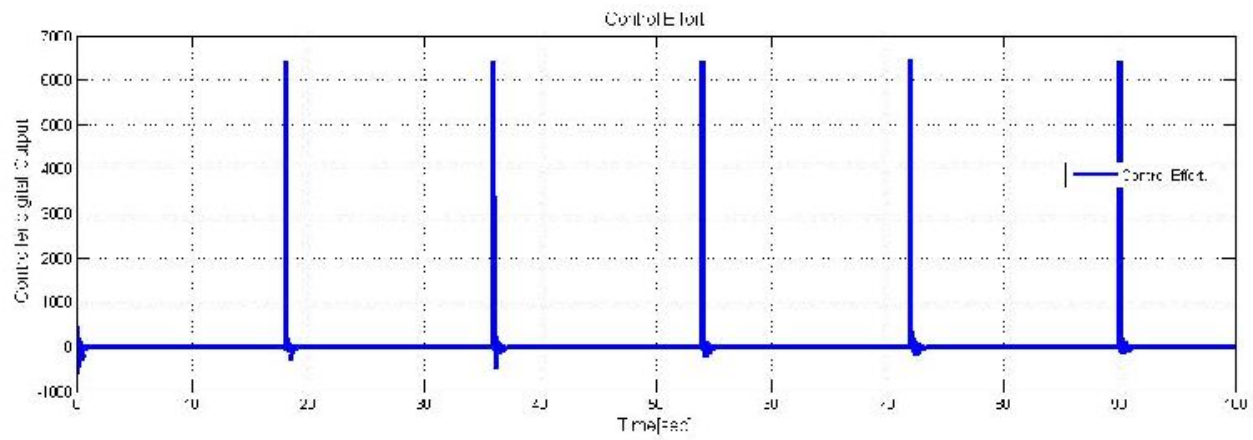

Fig.(18) Control Effort

\section{Conclusions and Future work}

In this paper, we describe the design process of improved PID controller in detail, and give an analysis of the efficiency of the control model in dynamic characteristics of tracking and eliminating the load disturbance. Experiments show that this controller can satisfy the target, and have a good performance and strong anti-interference ability. Although that advanced control theory provides many new control techniques, but the classical controller is still used and produces good performance concerning tracking, disturbance rejection and measurement noise and overcome the unmolded dynamics. However, several industrial application have the "dead time effect" due to the measurement delay or due to approximation of high order dynamic of the process by a transfer function model, and the classical controller fails to overcome these problems, which is the objective for future work also the controller necessitates extra tuning to become less sensitive to noises, which also is the objective for future work.

\section{Reference}


1. Johns Hopkins APL Technical Digest, VOLUME 29, NUMBER 1 (2010).

2. John Wiley \& Sons, Kalman Filtering Theory and Practice Using MATLAB, Third Edition,( 2008).

3. El-Sheikh, G.A., Advanced Guidance System, MTC, Cairo, 2010.

4. El-Sheikh, G.A., Guidance and Control, MTC, Cairo, 2010.

5. Ogata, K., Modern Control Engineering, Third Edition, Prentice Hall, 1997. 